International Journal of Applied Mathematics

Volume 33 No. $6 \quad 2020,969-994$

ISSN: 1311-1728 (printed version); ISSN: 1314-8060 (on-line version)

doi: http://dx.doi.org/10.12732/ijam.v33i6.2

\title{
ON A FRACTIONAL q-INTEGRAL OPERATOR INVOLVING THE BASIC ANALOGUE OF FOX-WRIGHT FUNCTION
}

\author{
Jaime Castillo $^{1}$, Leda Galué ${ }^{2}$ \\ ${ }^{1}$ Researching Center, University of La Guajira \\ Faculty of Engineering, Block 6 \\ Riohacha - 440002, COLOMBIA \\ ${ }^{2}$ CIMA, University of Zulia \\ Maracaibo - 4001, VENEZUELA
}

\begin{abstract}
The fractional $q$-calculus is the $q$-extension of the ordinary fractional calculus. In the second half of the twentieth century there was a significant increase of activity in the area of the q-calculus due to its applications in mathematics and physics. Particularly, the operators of fractional calculus and their $q$-analogues have many applications. They can be used in crack problems in elasticity, in chaos theory, control systems, signal processing, bio-medical engineering, radars, sonars, etc. The aim of this paper is to define a new fractional $q$-integral operator of Kober type using the basic analogue of Fox-Wright function as kernel, and to establish a composition formula for a particular type of these operators. Also, some particular cases are obtained.
\end{abstract}

AMS Subject Classification: 05A30, 33D05, 33D15, 47B33, 45P05

Key Words: $q$-fractional calculus; fractional $q$-integral operator; basic analogue of Fox-Wright function and composition of operators

\section{Introduction}

The fractional calculus treats the calculus of integrals and derivatives of any arbitrary real or complex order. It is almost as old as calculus itself [31], and it has received much attention due to its ability to model many natural phenomena $[24,30]$, and to solve a lot of problems in sciences, such as physics,

Received: October 2, 2020

(C) 2020 Academic Publications

${ }^{\S}$ Correspondence author 
electromagnetics, acoustics, viscoelasticity, electrochemistry and material science [32]. Further, for mathematics itself it provides new possibilities such as it is showed in $[12,23,53]$.

The fractional $q$-calculus is the $q$-extension of the ordinary fractional calculus. The first formulae in what we now call $q$-calculus were obtained by Euler in the eighteenth century. Many remarkable results (like Jacobi's triple product identity and the theory of $q$-hypergeometric functions) were obtained in the nineteenth century. In 1910, Jackson [26] introduced the notion of the definite $q$-integral, and he also was the first to develop $q$-calculus in a systematic way. In the second half of the twentieth century there was a significant increase of activity in the area of the $q$-calculus due to its applications in mathematics and physics, [22]. The treatment of the fractional calculus from the point of view of $q$-calculus can open new perspectives, [5]. Particularly, the $q$-analysis has found many applications in the theory of partitions, combinatorics, exactly solvable models in statistical mechanics, computer algebra, geometric functions theory, optimal control problems, $q$-difference and $q$-integral equations [3, 4, 21, 27, 29].

The operators of fractional calculus and their $q$-analogues have many applications, for example, they can be used to solve dual integral equations which arise in crack problems in elasticity [45]. They find applications also in chaos theory [50, 53, 54], control systems, signal processing, bio-medical engineering, radars, sonars, etc. $[9,48,49]$. By applying the fractional $q$-integral operators, many researchers have obtained a lot of fractional $q$-integral inequalities and applications $[6,7,8,10,18,34,35,43]$.

Rajkovic et al. [38] consider that the fractional calculus is a very suitable tool in describing and solving a lot of problems in sciences, such as physics, electromagnetics, acoustics, viscoelasticity, electrochemistry and material science (see, for example [32]). Of course, for mathematics itself it provides new possibilities such as it is emphasized in $[12,23,53]$. Their treatment from the point of view of $q$-calculus can open new perspectives (for example, see [5]). Another important property of fractional integrals is that certain multiples of them map some orthogonal polynomials to orthogonal polynomials, [25].

In this paper a new fractional $q$-integral operator of Kober type using the basic analogue of Fox-Wright function as kernel is defined, and a composition formula for a particular type of this operators is given. Also, some particular cases are obtained. 


\section{Some operators on fractional and q-fractional calculus}

The concept of differ-integral of complex order $\alpha$, which is a generalization of the ordinary $n$-th derivative and $n$ times integral to any complex number, can be introduced in several ways. The most widely used definition of an integral of fractional order is via an integral transform, called the Riemann-Liouville operator of fractional integration, [39]:

$$
\begin{gathered}
{ }_{a} I_{x}^{\alpha} f(x)=\frac{1}{\Gamma(\alpha)} \int_{a}^{x}(x-t)^{\alpha-1} f(t) d t, \quad \operatorname{Re}(\alpha)>0, \\
{ }_{a} D_{x}^{\alpha} f(x)=\frac{d^{n}}{d x^{n}}{ }_{a} I_{x}^{n-\alpha} f(x), \quad \operatorname{Re}(\alpha)>0, n=[\operatorname{Re}(\alpha)]+1 .
\end{gathered}
$$

Many authors $[1,2,11,12,13,14,15,16,17,18,19,20,28,33,37,38,40,41$, $42,51,52]$ have defined and studied operators of fractional integration through an integral transform.

\subsection{Erdélyi-Kober operator of fractional integration}

This operator of fractional order $\alpha(\operatorname{Re}(\alpha)>0)$, is introduced first by Kober and Erdélyi, and further developed by Sneddon [45] in the form

$$
\begin{gathered}
I_{\eta, \alpha} f(x)=\frac{x^{-(\alpha+\eta)}}{\Gamma(\alpha)} \int_{0}^{x}(x-t)^{\alpha-1} t^{\eta} f(t) d t \\
=x^{-(\alpha+\eta)} \frac{d^{n}}{d x^{n}} x^{\eta+\alpha+n} I_{\eta, \alpha+n} f(x), \quad \text { for } \operatorname{Re}(\alpha)<0, n>-\operatorname{Re}(\alpha),
\end{gathered}
$$

and has found very wide applications. See also Kiryakova [28].

\subsection{Basic analogues of the Riemann-Liouville fractional integral operator}

Agarwal [1] defined a basic analogue of the Riemann-Liouville fractional integral operator in the following form:

$$
I_{q}^{\alpha} f(x)=\frac{1}{\Gamma_{q}(\alpha)} \int_{0}^{x}(x-t q)_{\alpha-1} f(t) d_{q} t, \quad R e(\alpha)>0
$$

where $\alpha$ is an arbitrary order of integration, and

$$
(x-y)_{v} \equiv x^{v}\left(\frac{y}{x} ; q\right)_{v}=x^{v} \prod_{k=0}^{\infty}\left[\frac{1-\left(\frac{y}{x}\right) q^{k}}{1-\left(\frac{y}{x}\right) q^{k+v}}\right] .
$$


In 2012 Sulaiman [47] presented the following basic analogue of the RiemannLiouville fractional integral operator:

$$
J_{q}^{\alpha} f(x)=\frac{1}{\Gamma_{q}(\alpha)} \int_{0}^{t}(t-x)^{(\alpha-1)} f(x) d_{q} t, \quad \operatorname{Re}(\alpha)>0,
$$

where

$$
(t-x)^{(n)}= \begin{cases}(t-x)(t-q x) \ldots\left(t-q^{n-1} x\right), & \text { if } n \in \mathbb{Z}^{+} \\ t^{n} \prod_{k=0}^{\infty}\left[\frac{1-\left(\frac{x}{t}\right) q^{k}}{1-\left(\frac{x}{t}\right) q^{k+n}}\right], & \text { if } n \notin \mathbb{Z}^{+} .\end{cases}
$$

\subsection{Basic analogue of Kober fractional $q$-integral operator}

A basic analogue of the Kober fractional $q$-integral operator has been defined by Agarwal [1] in the following form:

$$
I_{q}^{\eta, \alpha} f(x)=\frac{x^{-(\eta+\alpha)}}{\Gamma_{q}(\alpha)} \int_{0}^{x} t^{\eta}(x-t q)_{\alpha-1} f(t) d_{q} t,
$$

provided $\eta, \alpha \in \mathbb{C}, \operatorname{Re}(\alpha)>0,|q|<1$.

\subsection{The operator $L_{q}\{\cdot\}$}

This operator was introduced in 2008 by Delgado and Galué [11] as follows:

$$
\begin{gathered}
L_{q}\left\{l, b_{1}, b_{2}, \ldots, b_{r}, \gamma, m_{1}, m_{2}, \ldots, m_{r} ; f(x)\right\}=\frac{x^{-\gamma-1}}{\Gamma_{q}(l+1)} \\
\times \int_{0}^{x} t^{\gamma}{ }_{r+1} \phi_{r}\left[\begin{array}{l}
q^{-l}, q^{m_{1}+b_{1}}, \ldots, q^{m_{r}+b_{r}} \\
q^{b_{1}}, \ldots, q^{b_{r}}
\end{array} q, q \frac{t}{x}\right] f(t) d_{q} t, \\
l, m_{1}, m_{2}, \ldots, m_{r} \in \mathbb{N}_{0}, \gamma \in \mathbb{C}, b_{1}, b_{2}, \ldots, b_{r} \neq 0,-1,-2, \ldots,\left|\frac{t}{x}\right|<1 .
\end{gathered}
$$

\subsection{The operator $L_{q}^{n}(\cdot)$}

In 2010 Galué [15] considered an $q$-fractional integral operator involving the basic analogue of the generalized hypergeometric function in the following form:

$$
L_{q}^{n}\left\{M, b_{1}, b_{2}, \ldots, b_{r}, \gamma, m_{1}, m_{2}, \ldots, m_{r} ; f(x)\right\}
$$




$$
\begin{gathered}
=\frac{x^{-\gamma-1}}{\Gamma_{q}(M+1)} \int_{0}^{x} t_{r+1}^{\gamma} \phi_{r}\left[\begin{array}{l}
q^{-M}, q^{b_{1}+m_{1}}, \ldots, q^{b_{r}+m_{r}} \\
q^{b_{1}}, \ldots, q^{b_{r}}
\end{array} ; q, q^{n} \frac{t}{x}\right] f(t) d_{q} t \\
M, m_{1}, m_{2}, \ldots, m_{r} \in \mathbb{N}_{0}, n \in \mathbb{N}, \gamma \in \mathbb{C} \\
b_{1}, b_{2}, \ldots, b_{r} \neq 0,-1,-2, \ldots,\left|\frac{t}{x}\right|<1,
\end{gathered}
$$

with a representation in series given by

$$
\begin{gathered}
L_{q}^{n}\left\{M, b_{1}, b_{2}, \ldots, b_{r}, \rho, m_{1}, m_{2}, \ldots, m_{r} ; f(x)\right\}= \\
\frac{(1-q)}{\Gamma_{q}(M+1)} \sum_{k=0}^{\infty} t^{(\rho+1) k}{ }_{r+1} \phi_{r}\left[\begin{array}{l}
q^{-M}, q^{b_{1}+m_{1}}, \ldots, q^{b_{r}+m_{r}} \\
q^{b_{1}}, \ldots, q^{b_{r}}
\end{array} ;, q^{n+k}\right] f\left(x q^{k}\right), \\
M, m_{1}, \ldots, m_{r} \in \mathbb{N}_{0}, n \in \mathbb{N}, \rho \in \mathbb{C}, b_{1}, b_{2}, \ldots, b_{r} \neq 0,-1,-2, \ldots
\end{gathered}
$$

\section{Preliminaries}

In this section we present some definitions, $q$-analogues of functions and known results needed in this paper.

Definition 1. If $q \in \mathbb{R}$ is fixed, a subset $A$ of $\mathbb{C}$ is called $q$-geometric if $q z \in A$ whenever $z \in A,[4]$.

Definition 2. A function $f$ which is defined on a $q$-geometric set $A, 0 \in A$, is said to be $q$-regular at zero if $\lim _{n \rightarrow \infty} f\left(z q^{n}\right)=f(0)$ for all $z \in A$, [4].

Definition 3. A function $f$ defined on $[0, a]$ is called $q$-absolutely continuous, that is $f \in \mathcal{A C}_{q}[0, a]$, if $f$ is $q$-regular at zero, and there exists $K>0$ such that $\sum_{j=0}^{\infty}\left|f\left(t q^{j}\right)-f\left(t q^{j+1}\right)\right| \leq K$ for all $t \in[q a, a],[4]$.

\section{1. $q$-Functions}

In this section some necessary preliminaries on the $q$-functions are presented. 


\subsubsection{Basic analogue of the $q$-shifted factorial.}

For real or complex $a$ and $0<|q|<1$, the $q$-factorial is defined as, [44]:

$$
(a ; q)_{n} \equiv\left(q^{a} ; q\right)_{n}= \begin{cases}1, & n=0 \\ \left(1-q^{a}\right)\left(1-q^{a+1}\right) \ldots\left(1-q^{a+n-1}\right), & n=1,2, \ldots\end{cases}
$$

Also, [21],

$$
(a ; q)_{\infty}=\lim _{x \rightarrow \infty}(a ; q)_{n}=\prod_{k=0}^{\infty}\left(1-a q^{k}\right),
$$

which converges for $|q|<1$ and diverges for $a \neq 0$ and $|q| \geq 1$, and for any complex number $\alpha$,

$$
(a ; q)_{\alpha}=\frac{(a ; q)_{\infty}}{\left(a q^{\alpha} ; q\right)_{\infty}}
$$

\subsubsection{Basic analogue of the gamma function.}

In [21], the basic analogue of the gamma function is defined in the following form:

$$
\Gamma_{q}(x)=\frac{(q ; q)_{\infty}}{\left(q^{x} ; q\right)_{\infty}}(1-q)^{1-x}=\frac{(q ; q)_{x-1}}{(1-q)^{x-1}}, 0<q<1, x \neq 0,-1,-2, \ldots .
$$

\subsubsection{Basic hypergeometric series.}

This series was introduced by Heine [21], and is defined as follows:

$$
{ }_{2} \phi_{1}(a, b ; c ; q, z)=\sum_{n=0}^{\infty} \frac{(a ; q)_{n}(b ; q)_{n}}{(q ; q)_{n}(c ; q)_{n}} z^{n},
$$

where it is assumed that $c \neq q^{-m}$ for $m=0,1, \ldots$, and $(a ; q)_{n}$ is the $q$-shifted factorial defined in (11).

\subsubsection{Generalized basic hypergeometric series.}

A generalization of the basic hypergeometric series ${ }_{2} \phi_{1}$, is given by [21]:

$$
\begin{gathered}
{ }_{r} \phi_{s}\left(a_{1}, a_{2}, \ldots, a_{r} ; b_{1}, b_{2}, \ldots, b_{s} ; q, z\right) \\
=\sum_{n=0}^{\infty} \frac{\left(a_{1} ; q\right)_{n}\left(a_{2} ; q\right)_{n} \cdots\left(a_{r} ; q\right)_{n}}{(q ; q)_{n}\left(b_{1} ; q\right)_{n} \cdots\left(b_{s} ; q\right)_{n}}\left[(-1)^{n} q^{\left.\left(\begin{array}{c}
n \\
2
\end{array}\right)\right]^{1+s-r}} z^{n},\right.
\end{gathered}
$$


where $b_{1}, \ldots, b_{s} \neq q^{-m}$ for $m=0,1, \ldots ;\left(\begin{array}{c}n \\ 2\end{array}\right)=n(n-1) / 2 ; q \neq 0$ when $r>$ $s+1$ and $\lim _{q \rightarrow 1^{-}}{ }_{r} \phi_{s}={ }_{r} F_{s}$. The ${ }_{r} \phi_{s}$-series terminates if one of its numerator parameters is of the form $q^{-m}$ with $m=0,1,2, \ldots$, and $q \neq 0$, since $\left(q^{-m} ; q\right)_{n}=$ 0 for $n=m+1, m+2, \ldots$.

\subsubsection{Basic analogue of the Fox-Wright function.}

Recently, Annaby and Mansour [4] introduced the following basic analogue of the Fox-Wright function:

$$
\begin{gathered}
{ }_{r} \psi_{s}\left[\begin{array}{c}
\left(\alpha_{1}, A_{1}\right), \ldots,\left(\alpha_{r}, A_{r}\right) \\
\left(\beta_{1}, B_{1}\right), \ldots,\left(\beta_{s}, B_{s}\right)
\end{array} \mid q, z\right]=\sum_{k=0}^{\infty} \frac{\prod_{i=1}^{r} \Gamma_{q}\left(\alpha_{i}+A_{i} k\right)}{\prod_{j=1}^{s} \Gamma_{q}\left(\beta_{j}+B_{j} k\right)} \\
\times\left[q^{\left(\begin{array}{c}
k \\
2
\end{array}\right)}\right]^{\sum_{j=1}^{s} B_{j}-\sum_{i=1}^{r} A_{i}+1} \frac{z^{k}}{\Gamma_{q}(k+1)},
\end{gathered}
$$

where $\Gamma_{q}(x)$ is the basic analogue of the gamma function as defined in (14).

In this paper we consider (17) with the following conditions: $A_{i}, B_{j} \in$ $\mathbb{R}^{+}, \operatorname{Re}\left(\alpha_{i}\right)>0, \operatorname{Re}\left(\beta_{j}\right)>0, i=1, \ldots, r, j=1, \ldots, s, \sum_{j=1}^{s} B_{j}-\sum_{i=1}^{r} A_{i}+$ $1 \geq 0,0<q<1,|z|<1$.

Using (14) and (13) in (17), we can write

$$
\begin{aligned}
& { }_{r} \psi_{s}\left[\begin{array}{c}
\left(\alpha_{1}, A_{1}\right), \ldots,\left(\alpha_{r}, A_{r}\right) \\
\left(\beta_{1}, B_{1}\right), \ldots,\left(\beta_{s}, B_{s}\right)
\end{array} \mid q, z\right]=\frac{\prod_{i=1}^{r} \Gamma_{q}\left(\alpha_{i}\right)}{\prod_{j=1}^{s} \Gamma_{q}\left(\beta_{j}\right)}
\end{aligned}
$$

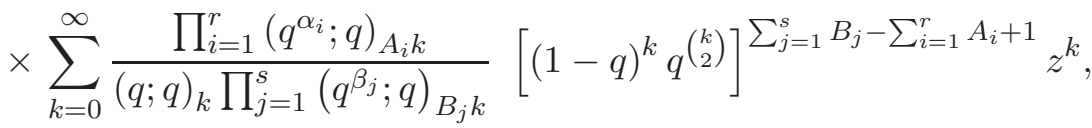

$$
\begin{aligned}
& A_{i}, B_{j} \in \mathbb{R}^{+}, \operatorname{Re}\left(\alpha_{i}\right)>0, \operatorname{Re}\left(\beta_{j}\right)>0, i=1, \ldots, r, j=1, \ldots, s, \\
& \sum_{j=1}^{s} B_{j}-\sum_{i=1}^{r} A_{i}+1 \geq 0,0<q<1,|z|<1 .
\end{aligned}
$$

The normalized form of (18) is given by

$$
\begin{gathered}
\left.{ }_{r} \psi_{s}^{*}\left[\begin{array}{c}
\left(\alpha_{1}, A_{1}\right), \ldots,\left(\alpha_{r}, A_{r}\right) \\
\left(\beta_{1}, B_{1}\right), \ldots,\left(\beta_{s}, B_{s}\right)
\end{array}\right) q, z\right]=\sum_{k=0}^{\infty} \frac{\prod_{i=1}^{r}\left(q^{\alpha_{i}} ; q\right)_{A_{i} k}}{(q ; q)_{k} \prod_{j=1}^{s}\left(q^{\beta_{j}} ; q\right)_{B_{j} k}} \\
\times\left[(1-q)^{k} q^{\left(\begin{array}{c}
k \\
2
\end{array}\right)}\right]^{\sum_{j=1}^{s} B_{j}-\sum_{i=1}^{r} A_{i}+1} z^{k},
\end{gathered}
$$

$A_{i}, B_{j} \in \mathbb{R}^{+}, \operatorname{Re}\left(\alpha_{i}\right)>0, \operatorname{Re}\left(\beta_{j}\right)>0, i=1, \ldots, r, j=1, \ldots, s$, 


$$
\sum_{j=1}^{s} B_{j}-\sum_{i=1}^{r} A_{i}+1 \geq 0,|z|<1 .
$$

\section{Particular Cases:}

Case 1: In (17) taking $q \rightarrow 1^{-}$, we have as particular case the hypergeometric function of Fox-Wright (see for example, [46]).

Case 2: Taking $A_{r}=1$ and $\alpha_{r}=-M, M \in \mathbb{N}_{0}$, in (19), we have

$$
\begin{aligned}
& { }_{r} \psi_{s}^{*}\left[\begin{array}{l|l}
\left(\alpha_{1}, A_{1}\right), \ldots,\left(\alpha_{r-1}, A_{r-1}\right),(-M, 1) & q, z \\
\left(\beta_{1}, B_{1}\right), \ldots,\left(\beta_{s}, B_{s}\right)
\end{array}\right] \\
& =\sum_{k=0}^{\infty} \frac{\left(q^{-M} ; q\right)_{k} \prod_{i=1}^{r-1}\left(q^{\alpha_{i}} ; q\right)_{A_{i} k}}{(q ; q)_{k} \prod_{j=1}^{s}\left(q^{\beta_{j}} ; q\right)_{B_{j} k}}\left[(1-q)^{k} q^{\left(\begin{array}{c}
k \\
2
\end{array}\right)}\right]^{\sum_{j=1}^{s} B_{j}-\sum_{i=1}^{r-1} A_{i}} z^{k}, \\
& A_{i}, B_{j} \in \mathbb{R}^{+}, \operatorname{Re}\left(\alpha_{i}\right)>0, \operatorname{Re}\left(\beta_{j}\right)>0, i=1, \ldots, r-1, j=1, \ldots, s, \\
& M \in \mathbb{N}_{0}, \sum_{j=1}^{s} B_{j}-\sum_{i=1}^{r-1} A_{i} \geq 0,0<q<1,|z|<1 .
\end{aligned}
$$

Case 3: For $r=s+1$ in (20) we obtain $i=j$ and then for $A_{j}=B_{j}=1$ results

$$
\begin{gathered}
{ }_{s+1} \psi_{s}^{*}\left[\begin{array}{l}
\left(\alpha_{1}, 1\right), \ldots,\left(\alpha_{s}, 1\right),(-M, 1) \\
\left(\beta_{1}, 1\right), \ldots,\left(\beta_{s}, 1\right)
\end{array} \mid q, z\right] \\
=\sum_{k=0}^{M} \frac{\left(q^{-M} ; q\right)_{k}}{(q ; q)_{k}} \frac{\prod_{j=1}^{s}\left(q^{\alpha_{j}} ; q\right)_{k}}{\prod_{j=1}^{s}\left(q^{\beta_{j}} ; q\right)_{k}} z^{k} \\
={ }_{s+1} \phi_{s}\left(q^{-M}, q^{\alpha_{1}}, \ldots, q^{\alpha_{s}}, q^{\beta_{1}}, \ldots, q^{\beta_{s}} ; q, z\right),
\end{gathered}
$$

$\alpha_{j} \in \mathbb{C}, \operatorname{Re}\left(\beta_{j}\right) \neq 0,-1,-2, \ldots ; j=1, \ldots, s, M \in \mathbb{N}_{0}, 0<q<1,|z|<1$.

The next theorem establishes the absolute convergence of the basic analogue of the Fox-Wright function, as we hope to be a new result.

Theorem 4. Let be $A_{i}, B_{j} \in \mathbb{R}^{+}, \operatorname{Re}\left(\alpha_{i}\right)>0, \operatorname{Re}\left(\beta_{j}\right)>0, i=$ $1, \ldots, r, j=1, \ldots, s, z \in \mathbb{C}$ and $0<q<1$.

i) If $\sum_{j=1}^{s} B_{j}-\sum_{i=1}^{r} A_{i}+1>0$, the series ${ }_{r} \psi_{s}\left[\begin{array}{c}\left(\alpha_{1}, A_{1}\right), \ldots,\left(\alpha_{r}, A_{r}\right) \\ \left(\beta_{1}, B_{1}\right), \ldots,\left(\beta_{s}, B_{s}\right)\end{array} \mid q, z\right]$ converges absolutely for all $z \in \mathbb{C}$.

ii) If $\sum_{j=1}^{s} B_{j}-\sum_{i=1}^{r} A_{i}+1<0, z \neq 0$, the series ${ }_{r} \psi_{s}\left[\begin{array}{cc}\left(\alpha_{1}, A_{1}\right), \ldots, & \left(\alpha_{r}, A_{r}\right) \\ \left(\beta_{1}, B_{1}\right), \ldots, & \left(\beta_{s}, B_{s}\right)\end{array} \mid q, z\right]$ diverges. 
iii) If $\sum_{j=1}^{s} B_{j}-\sum_{i=1}^{r} A_{i}+1=0$, the series ${ }_{r} \psi_{s}\left[\begin{array}{c}\left(\alpha_{1}, A_{1}\right), \ldots,\left(\alpha_{r}, A_{r}\right) \\ \left(\beta_{1}, B_{1}\right), \ldots,\left(\beta_{s}, B_{s}\right)\end{array} \mid q, z\right]$ converges absolutely for $|z|<1$.

The proof of this theorem follows in straight way from (18) applying the ratio test.

\subsection{Known Results}

\subsubsection{Identities.}

The following $q$-identities are given in [21], page 6: eqs. (1.2.33), (1.2.35), $(1.2 .38)$ :

$$
\begin{gathered}
(a ; q)_{n+k}=(a ; q)_{n}\left(a q^{n} ; q\right)_{k}, \\
\left(a q^{k} ; q\right)_{n-k}=\frac{(a ; q)_{n}}{(a ; q)_{k}}, \\
\left(a q^{-n} ; q\right)_{k}=\frac{(a ; q)_{k}\left(q a^{-1} ; q\right)_{n}}{\left(a^{-1} q^{1-k} ; q\right)_{n}} q^{-n k} .
\end{gathered}
$$

In 2010, Galué [15] presented the following identities:

$$
\begin{gathered}
\sum_{k=0}^{\nu} q^{(y-w) k}=q^{(y-w) \nu} \frac{\left(q^{-y} ; q\right)_{w}}{\left(q^{1-y} ; q\right)_{w}\left(q^{-y} ; q\right)_{1}}, \\
\frac{\left(q^{\beta_{i 1}-1-M_{1}+j} ; q\right)_{m_{i 1}}}{\left(q^{\beta_{i 1}} ; q\right)_{m_{i 1}}} \\
=\frac{\left(q^{\beta_{i 1}+m_{i 1}-1-M_{1}} ; q\right)_{j} \Gamma_{q}\left(\beta_{i 1}+m_{i 1}-1-M_{1}\right) \Gamma_{q}\left(\beta_{i 1}\right)}{\left(q^{\beta_{i 1}-1-M_{1}} ; q\right)_{j} \Gamma_{q}\left(\beta_{i 1}+m_{i 1}\right) \Gamma_{q}\left(\beta_{i 1}-1-M_{1}\right)} \\
i=1, \ldots, s .
\end{gathered}
$$

\subsubsection{The $q$-integral.}

Jackson [26] introduced the $q$-integral as

$$
\int_{a}^{b} f(t) d_{q} t=\int_{0}^{b} f(t) d_{q} t-\int_{0}^{a} f(t) d_{q} t
$$

where

$$
\int_{0}^{a} f(t) d_{q} t=a(1-q) \sum_{n=0}^{\infty} f\left(a q^{n}\right) q^{n}
$$




\subsubsection{The $q$-binomial theorem.}

One of the most important summation formulas for hypergeometric series is given by the binomial theorem, whose $q$-analogue, derived by Cauchy (1843), Heine (1847) and other mathematicians, is [21]:

$$
{ }_{1} \phi_{0}\left[\begin{array}{c}
\alpha \\
-
\end{array} ;, x\right]=\sum_{n=0}^{\infty} \frac{(\alpha ; q)_{n}}{(q ; q)_{n}} x^{n}=\frac{(\alpha x ; q)_{\infty}}{(x ; q)_{\infty}},|x|<1,|q|<1 .
$$

\subsection{4. $q$-analogue of the Karlsson-Minton summation formula.}

Gasper [21] derived a $q$-analogue of the Karlsson-Minton summation formula, which is given by ([p.16, No. (1.9.10)]):

$$
\begin{array}{r}
{ }_{r+2} \phi_{r+1}\left[\begin{array}{l}
q^{-n}, b, b_{1} q^{m_{1}}, \ldots, b_{r} q^{m_{r}} ; q, q \\
b q, b_{1}, \ldots, b_{r}
\end{array}\right] \\
=\frac{b^{n}(q ; q)_{n}}{(b q ; q)_{n}} \frac{\left(b_{1} / b ; q\right)_{m_{1}} \ldots\left(b_{r} / b ; q\right)_{m_{r}}}{\left(b_{1} ; q\right)_{m_{1}} \ldots\left(b_{r} ; q\right)_{m_{r}}}
\end{array}
$$

for $n \geq m_{1}+\ldots+m_{r} ; m_{1}, m_{2}, \ldots, m_{r}$ nonnegative integers.

\section{The operator $I_{q}^{n}(\cdot)$}

In this section a new fractional $q$-integral operator, denoted by $I_{q}^{n}(\cdot)$ is defined.

Definition 5. Let be $f$ a $q$-absolutely continuous function on $[0, x]$, then we define the operator

$$
\begin{gathered}
I_{q}^{n}\left[\begin{array}{l}
\rho,(M, 1),\left(\alpha_{i}, A_{i}\right)_{1, r-1} \\
\left(\beta_{j}, B_{j}\right)_{1, s}
\end{array}\right] f(x)=\frac{x^{-\rho-1}}{\Gamma_{q}(M+1)} \times \\
\int_{0}^{x} t_{r}^{\rho} \psi_{s}^{*}\left[\begin{array}{l}
\left(\alpha_{1}, A_{1}\right), \ldots,\left(\alpha_{r-1}, A_{r-1}\right),(-M, 1) \\
\left(\beta_{1}, B_{1}\right), \ldots,\left(\beta_{s}, B_{s}\right)
\end{array} \mid q, q^{n} \frac{t}{x}\right] f(t) d_{q} t, \\
A_{i}, B_{j} \in \mathbb{R}^{+}, \operatorname{Re}\left(\alpha_{i}\right)>0, \operatorname{Re}\left(\beta_{j}\right)>0, i=1, \cdots, r-1 ; j=1, \cdots, s, \\
\sum_{j=1}^{s} B_{j}-\sum_{i=1}^{r-1} A_{i} \geq 0, M \in \mathbb{N}_{0}, n \in \mathbb{N}, \rho \in \mathbb{C}, 0<q<1,\left|\frac{t}{x}\right|<1 .
\end{gathered}
$$


By virtue of the result (28), the operator (31) can be written as

$$
\begin{gathered}
I_{q}^{n}\left[\begin{array}{l}
\rho,(M, 1),\left(\alpha_{i}, A_{i}\right)_{1, r-1} \\
\left(\beta_{j}, B_{j}\right)_{1, s}
\end{array}\right] f(x)=\frac{(1-q)}{\Gamma_{q}(M+1)} \sum_{k=0}^{\infty} q^{(\rho+1) k} \times \\
r \psi_{s}^{*}\left[\begin{array}{l}
\left(\alpha_{1}, A_{1}\right), \ldots,\left(\alpha_{r-1}, A_{r-1}\right),(-M, 1) \mid q, q^{n+k} \\
\left(\beta_{1}, B_{1}\right), \ldots,\left(\beta_{s}, B_{s}\right)
\end{array}\right] f\left(x q^{k}\right), \\
A_{i}, B_{j} \in \mathbb{R}^{+}, \operatorname{Re}\left(\alpha_{i}\right)>0, R e\left(\beta_{j}\right)>0, i=1, \ldots, r-1 ; j=1, \ldots, s, \\
\sum_{j=1}^{s} B_{j}-\sum_{i=1}^{r-1} A_{i} \geq 0, M \in \mathbb{N}_{0}, n \in \mathbb{N}, \rho \in \mathbb{C}, 0<q<1 .
\end{gathered}
$$

For convenience we will use the notation:

$$
I_{q}^{n}(\cdot) \equiv I_{q}^{n}\left[\begin{array}{l}
\rho,(M, 1),\left(\alpha_{i}, A_{i}\right)_{1, r-1} \\
\left(\beta_{j}, B_{j}\right)_{1, s}
\end{array}\right]
$$

\section{Particular Cases:}

Case 1. The operator $L_{q}^{n}(\cdot)$ was considered by Galué.

Taking $r=s+1, A_{i}=B_{i}=1, \alpha_{i}=\beta_{i}+m_{i}, m_{i} \in \mathbb{N}_{0} ; i=1, \ldots, s$ in (31), then according to (21) and using (9) we get

$$
\begin{gathered}
I_{q}^{n}\left[\begin{array}{l}
\rho,(-M, 1),\left(\beta_{i}+m_{i}, 1\right)_{1, s} \\
\left(\beta_{i}, 1\right)_{1, s}
\end{array}\right] f(x) \\
=L_{q}^{n}\left\{M, \beta_{1}, \ldots, \beta_{s}, \rho, m_{1}, \ldots, m_{s} ; f(x)\right\}, \\
m_{i}, M \in \mathbb{N}_{0}, \operatorname{Re}\left(\beta_{i}\right) \neq 0,-1,-2, \ldots, i=1, \ldots, s, n \in \mathbb{N}, \\
\rho \in \mathbb{C}, 0<q<1,\left|\frac{t}{x}\right|<1 .
\end{gathered}
$$

Case 2. Operator $L_{q}(\cdot)$ established by Delgado and Galué.

It is obtained directly from (33) making $n=1$.

If we consider $r=s+1, A_{i}=B_{i}, \alpha_{i}=\beta_{i}, i=1, \ldots, s, n=M+1$ in (31), then for $\left|\frac{t}{x}\right|<1$ :

$$
\begin{gathered}
I_{q}^{n}\left[\begin{array}{c}
\rho,(M, 1) \\
-
\end{array}\right] f(x)=\frac{x^{-\rho-1}}{\Gamma_{q}(M+1)} \int_{0}^{x} t^{\rho} \\
\times{ }_{1} \psi_{0}^{*}\left[\begin{array}{c}
(-M, 1) \\
-
\end{array} q, q^{n} \frac{t}{x}\right] f(t) d_{q} t, M \in \mathbb{N}_{0}, \rho \in \mathbb{C}, 0<q<1,
\end{gathered}
$$


and now, applying (21), (29), and (12) respectively, we obtain again for $\left|\frac{t}{x}\right|<1$ :

$$
\begin{gathered}
I_{q}^{n}\left[\begin{array}{c}
\rho,(M, 1) \\
-
\end{array}\right] f(x)=\frac{x^{-\rho-1}}{\Gamma_{q}(M+1)} \int_{0}^{x} t^{\rho} \\
\times \prod_{k=0}^{\infty}\left[\frac{\left(1-\left(q^{-M+n} \frac{t}{x}\right) q^{k}\right)}{1-\left(q^{-M+n} \frac{t}{x}\right) q^{k+M}}\right] f(t) d_{q} t, M \in \mathbb{N}_{0}, \rho \in \mathbb{C}, 0<q<1 .
\end{gathered}
$$

This expression will be used in the next results.

Case 3. Basic analogue of Kober fractional integral operator.

From (34) with $n=M+1$ it results

$$
I_{q}^{M+1}\left[\begin{array}{c}
\rho,(M, 1) \\
-
\end{array}\right] f(x)=\frac{x^{-\rho-M-1}}{\Gamma_{q}(M+1)} \int_{0}^{x} t^{\rho}(x-t q)_{M} f(t) d_{q} t,
$$

where we have used (4). Therefore from (7) we can write

$$
\begin{gathered}
I_{q}^{M+1}\left[\begin{array}{c}
\rho,(M, 1) \\
-
\end{array}\right] f(x)=I_{q}^{\rho, M+1} f(x), \\
M \in \mathbb{N}_{0}, \rho \in \mathbb{C}, 0<q<1,\left|\frac{t}{x}\right|<1 .
\end{gathered}
$$

Case 4. Basic analogues of Riemann-Liouville fractional integral operator.

4.1. From (35) with $\rho=0$ and using (3), we have

$$
\begin{gathered}
I_{q}^{M+1}\left[\begin{array}{c}
0, \\
-
\end{array}\right] f(x)=x^{-(M+1)} I_{q}^{M+1} f(x), \\
M \in \mathbb{N}_{0}, 0<q<1,\left|\frac{t}{x}\right|<1 .
\end{gathered}
$$

4.2. From (35) with $\rho=0, n=M$ and using (6) and later (5), we obtain

$$
\begin{gathered}
I_{q}^{M}\left[\begin{array}{c}
0, \\
-
\end{array}\right] f(x)=x^{-(M+1)} J_{q}^{M+1} f(x), \\
M \in \mathbb{N}, 0<q<1,\left|\frac{t}{x}\right|<1 .
\end{gathered}
$$




\subsection{Composition formulae for $I_{q}^{n}[\cdot]$ fractional $q$-integral operators.}

In this section we establish some formulae for the composition of the operator $q$ fractional integral $I_{q}^{n}(\cdot)$ with the operator $L_{q}\left\{M, b_{1}, b_{2}, \ldots, b_{s}, \rho, m_{1}, m_{2}, \ldots, m_{s}\right.$; $f(x)\}$, presented by Delgado and Galué in [11].

For convenience we will use the following notation:

$$
\begin{gathered}
L_{q}\left\{M, b_{11}, b_{21}, \ldots, b_{r 1}, \rho, m_{11}, m_{21}, \ldots, m_{r 1} ; f(x)\right\} \\
=L_{q}\left[\begin{array}{c}
M, b_{11}, b_{21}, \ldots, b_{r 1} \\
\rho, m_{11}, m_{21}, \ldots, m_{r 1}
\end{array}\right] f(x) .
\end{gathered}
$$

Theorem 6. Let $f$ be a $q$-absolutely continuous function on $[0, x]$,

$$
L_{q}\left[\begin{array}{c}
M, b_{12}, b_{22}, \ldots, b_{r 2} \\
\rho, m_{12}, m_{22}, \ldots, m_{r 2}
\end{array}\right](\cdot) \text { and } I_{q}^{n}\left[\begin{array}{l}
\rho,(M, 1),\left(\alpha_{i}, A_{i}\right)_{1, r-1} \\
\left(\beta_{j}, B_{j}\right)_{1, s}
\end{array}\right](\cdot)
$$

be fractional q-integral operators as defined by (8) and (31) respectively, then

$$
\begin{gathered}
L_{q}\left[\begin{array}{l}
M_{2}, b_{12}, b_{22}, \cdots, b_{s 2} \\
\rho+M_{1}+1, m_{12}, m_{22}, \cdots, m_{s 2}
\end{array}\right] \\
\times I_{q}^{n}\left[\begin{array}{l}
\left.\rho,\left(M_{1}, 1\right),\left(\alpha_{i}, A_{i}\right)_{1, r-1}\right] \\
\left(\beta_{j}, B_{j}\right)_{1, s}
\end{array}\right] f(x) \\
=q^{\left(1+M_{1}\right) M_{2}} \prod_{v=1}^{s}\left[\frac{\Gamma_{q}\left(b_{v 2}+m_{v 2}-1-M_{1}\right) \Gamma_{q}\left(b_{v 2}\right)}{\Gamma_{q}\left(b_{v 2}-1-M_{1}\right) \Gamma_{q}\left(b_{v 2}+m_{v 2}\right)}\right] \times \\
I_{q}^{n+1}\left[\begin{array}{l}
\rho,\left(M_{2}+M_{1}+1,1\right),\left(\alpha_{i}, A_{i}\right)_{1, r-1},\left(b_{j 2}+m_{j 2}-1-M_{1}, 1\right)_{1, s} \\
\left(\beta_{j}, B_{j}\right)_{1, s},\left(b_{j 2}-1-M_{1}, 1\right)_{1, s}
\end{array}\right] f(x), \\
A_{i}, B_{j} \in \mathbb{R}^{+}, R e\left(\alpha_{i}\right)>0, R e\left(\beta_{j}\right)>0, i=1, \ldots, r-1 ; j=1, \ldots, s, \\
M_{1}, M_{2}, m_{j 2} \in \mathbb{N}_{0}, n \in \mathbb{N}, \rho \in \mathbb{C}, 0<q<1, \sum_{j=1}^{s} B_{j}-\sum_{i=1}^{r-1} A_{i} \geq 0, \\
b_{j 2} \neq 1+M_{1}, M_{1}, M_{1}-1, \ldots, M_{2} \geq m_{12}+\ldots+m_{s 2} .
\end{gathered}
$$

Proof. On applying (10) with $n=1$ to (32), using the notations $L_{q}^{n}(\cdot)$, $I_{q}^{n}[\cdot]$ and the result $([36])$

$$
\sum_{n=0}^{\infty} \sum_{k=0}^{\infty} A(k, n)=\sum_{n=0}^{\infty} \sum_{k=0}^{n} A(k, n-k)
$$


we have

$$
\begin{gathered}
L_{q}(\cdot) I_{q}^{n}[\cdot] f(x)=\frac{(1-q)^{2}}{\Gamma_{q}\left(M_{1}+1\right) \Gamma_{q}\left(M_{2}+1\right)} \sum_{h=0}^{\infty} q^{\left(\rho+M_{1}+2\right) h} f\left(x q^{h}\right) \\
\times\left\{\begin{array}{l}
\sum_{k=0}^{h} q^{-\left(M_{1}+1\right) k}{ }_{s+1} \phi_{s}\left[\begin{array}{l}
q^{-M_{2}}, q^{b_{12}+m_{12}}, \ldots, q^{b_{s 2}+m_{s 2}} \\
q^{b_{12}}, \ldots, q^{b_{s 2}}
\end{array} ; q, q^{1+h-k}\right] \\
\left.\times{ }_{r} \psi_{s}^{*}\left[\begin{array}{l}
\left(\alpha_{1}, A_{1}\right), \ldots,\left(\alpha_{r-1}, A_{r-1}\right),\left(-M_{1}, 1\right) \\
\left(\beta_{1}, B_{1}\right), \ldots,\left(\beta_{s}, B_{s}\right)
\end{array} \mid q, q^{n+k}\right]\right\} .
\end{array}\right.
\end{gathered}
$$

In view of the results (16) and (20) we can write

$$
\begin{aligned}
& L_{q}(\cdot) I_{q}^{n}[\cdot] f(x)=\frac{(1-q)^{2}}{\Gamma_{q}\left(M_{1}+1\right) \Gamma_{q}\left(M_{2}+1\right)} \sum_{h=0}^{\infty} q^{\left(\rho+M_{1}+2\right) h} f\left(x q^{h}\right) \\
& \times \sum_{k=0}^{h} q^{-\left(M_{1}+1\right) k}\left\{\sum_{w=0}^{\infty} \frac{\left(q^{-M_{2}}, q^{b_{12}+m_{12}}, \ldots, q^{b_{s 2}+m_{s 2}} ; q\right)_{w}}{\left(q, q^{b_{12}}, \ldots, q^{b_{s 2}} ; q\right)_{w}} q^{(1+h-k) w}\right\} \\
& \times\left\{\sum_{p=0}^{\infty} \frac{\left(q^{-M_{1}} ; q\right)_{p} \prod_{u=1}^{r-1}\left(q^{\alpha_{u}} ; q\right)_{A_{u} p}}{(q ; q)_{p} \prod_{u=1}^{s}\left(q^{\beta_{u}} ; q\right)_{B_{u} p}}\right\} \\
& \times\left[(1-q)^{p} q^{\left.\left.\left(\begin{array}{c}
p \\
2
\end{array}\right)\right]^{\sum_{u=1}^{s} B_{u}-\sum_{u=1}^{r-1} A_{u}} q^{(n+k) p}\right\},}\right.
\end{aligned}
$$

where from [21] [p.6, eq. (1.2.41)]:

$$
\left(a_{1}, a_{2}, \ldots, a_{m} ; q\right)_{n}=\left(a_{1} ; q\right)_{n}\left(a_{2} ; q\right)_{n} \ldots\left(a_{m} ; q\right)_{n}
$$

Interchanging the order of the series, on the basis of the absolute convergence, we get

$$
\begin{aligned}
& L_{q}(\cdot) I_{q}^{n}[\cdot] f(x)=\frac{(1-q)^{2}}{\Gamma_{q}\left(M_{1}+1\right) \Gamma_{q}\left(M_{2}+1\right)} \sum_{h=0}^{\infty} q^{\left(\rho+M_{1}+2\right) h} f\left(x q^{h}\right) \times \\
& \sum_{p=0}^{\infty} \frac{\left(q^{-M_{1}} ; q\right)_{p} \prod_{u=1}^{r-1}\left(q^{\alpha_{u}} ; q\right)_{A_{u} p}}{(q ; q)_{p} \prod_{u=1}^{s}\left(q^{\beta_{u}} ; q\right)_{B_{u} p}}\left[(1-q)^{p} q^{\left(\begin{array}{c}
p \\
2
\end{array}\right)}\right]^{\sum_{u=1}^{s} B_{u}-\sum_{u=1}^{r-1} A_{u}} q^{n p} \\
& \times\left\{\sum_{w=0}^{\infty} \frac{\left(q^{-M_{2}}, q^{b_{12}+m_{12}}, \ldots, q^{b_{s 2}+m_{s 2}} ; q\right)_{w}}{\left(q, q^{b_{12}}, \ldots, q^{b_{s 2}} ; q\right)_{w}} q^{(1+h) w}\left[\sum_{k=0}^{h} q^{\left(p-w-M_{1}-1\right) k}\right]\right\} .
\end{aligned}
$$


From (25),

$$
\begin{aligned}
& L_{q}(\cdot) I_{q}^{n}[\cdot] f(x)=\frac{(1-q)^{2}}{\Gamma_{q}\left(M_{1}+1\right) \Gamma_{q}\left(M_{2}+1\right)} \sum_{h=0}^{\infty} q^{\left(\rho+M_{1}+2\right) h} f\left(x q^{h}\right) \\
& \times \sum_{p=0}^{\infty} \frac{\left(q^{-M_{1}} ; q\right)_{p} \prod_{u=1}^{r-1}\left(q^{\alpha_{u}} ; q\right)_{A_{u} p}}{(q ; q)_{p} \prod_{u=1}^{s}\left(q^{\beta_{u}} ; q\right)_{B_{u} p}} \\
& \times\left[(1-q)^{p} q^{\left(\begin{array}{l}
p \\
2
\end{array}\right)}\right]^{\sum_{u=1}^{s} B_{u}-\sum_{u=1}^{r-1} A_{u}} q^{n p} \\
& \times\left\{\sum_{w=0}^{\infty} \frac{\left(q^{-M_{2}}, q^{b_{12}+m_{12}}, \ldots, q^{b_{s 2}+m_{s 2}} ; q\right)_{w}}{\left(q, q^{b_{12}}, \ldots, q^{b_{s 2}} ; q\right)_{w}} q^{(1+h) w}\right. \\
& \left.\times \frac{\left(q^{1-p+M_{1}} ; q\right)_{w} q^{\left(p-w-M_{1}-1\right) h}}{\left(q^{2-p+M_{1}} ; q\right)_{w}\left(q^{1-p+M_{1}} ; q\right)_{1}}\right\},
\end{aligned}
$$

that is,

$$
\begin{aligned}
& L_{q}(\cdot) I_{q}^{n}[\cdot] f(x)=\frac{(1-q)^{2}}{\Gamma_{q}\left(M_{1}+1\right) \Gamma_{q}\left(M_{2}+1\right)} \sum_{h=0}^{\infty} q^{(\rho+1) h} f\left(x q^{h}\right) \\
& \times\left\{\sum_{p=0}^{\infty} \frac{\left(q^{-M_{1}} ; q\right)_{p} \prod_{u=1}^{r-1}\left(q^{\alpha_{u}} ; q\right)_{A_{u} p}}{(q ; q)_{p}\left(q^{1-p+M_{1}} ; q\right)_{1} \prod_{u=1}^{s}\left(q^{\beta_{u}} ; q\right)_{B_{u} p}}\right. \\
& \left.\times\left[(1-q)^{p} q^{\left(\begin{array}{c}
p \\
2
\end{array}\right)}\right]^{\sum_{u=1}^{s} B_{u}-\sum_{u=1}^{r-1} A_{u}} q^{(n+h) p}\right\} \\
& \times_{s+2} \phi_{s+1}\left[\begin{array}{ll}
q^{-M_{2}}, q^{1-p+M_{1}}, q^{b_{12}+m_{12}}, \ldots, q^{b_{s 2}+m_{s 2}} & ; q, q \\
q^{2-p+M_{1}}, q^{b_{12}}, \ldots, q^{b_{s 2}} &
\end{array}\right],
\end{aligned}
$$

where we have used (16).

Now, the formula (30) leads us to

$$
\begin{aligned}
& L_{q}(\cdot) I_{q}^{n}[\cdot] f(x)=\frac{(1-q)^{2}}{\Gamma_{q}\left(M_{1}+1\right) \Gamma_{q}\left(M_{2}+1\right)} \sum_{h=0}^{\infty} q^{(\rho+1) h} f\left(x q^{h}\right) \\
& \times\left\{\sum_{p=0}^{\infty} \frac{\left(q^{-M_{1}} ; q\right)_{p} \prod_{u=1}^{r-1}\left(q^{\alpha_{u}} ; q\right)_{A_{u} p}}{(q ; q)_{p}\left(q^{1-p+M_{1}} ; q\right)_{1} \prod_{u=1}^{s}\left(q^{\beta_{u}} ; q\right)_{B_{u} p}}\right. \\
& \left.\left[(1-q)^{p} q^{\left(\begin{array}{l}
p \\
2
\end{array}\right)}\right]^{\sum_{u=1}^{s} B_{u}-\sum_{u=1}^{r-1} A_{u}} q^{(n+h) p}\right\} \times
\end{aligned}
$$




$$
\frac{q^{\left(1-p+M_{1}\right) M_{2}}(q ; q)_{M_{2}}}{\left(q^{2-p+M_{1}} ; q\right)_{M_{2}}} \frac{\left(q^{b_{12}-1-M_{1}+p} ; q\right)_{m_{12}} \ldots\left(q^{b_{s 2}-1-M_{1}+p} ; q\right)_{m_{s 2}}}{\left(q^{b_{12}} ; q\right)_{m_{12}} \ldots\left(q^{b_{s 2}} ; q\right)_{m_{s 2}}}
$$

with $M_{2} \geq m_{12}+\ldots+m_{s 2}$.

On using (22) and (26), we can write (43) in the following form:

$$
\begin{aligned}
& L_{q}(\cdot) I_{q}^{n}[\cdot] f(x) \\
& =\frac{(1-q)^{2}(q ; q)_{M_{2}} q^{\left(1+M_{1}\right) M_{2}}}{\Gamma_{q}\left(M_{1}+1\right) \Gamma_{q}\left(M_{2}+1\right)} \prod_{v=1}^{s}\left[\frac{\Gamma_{q}\left(b_{v 2}+m_{v 2}-1-M_{1}\right)}{\Gamma_{q}\left(b_{v 2}-1-M_{1}\right)}\right. \\
& \left.\times \frac{\Gamma_{q}\left(b_{v 2}\right)}{\Gamma_{q}\left(b_{v 2}+m_{v 2}\right)}\right] \sum_{h=0}^{\infty} q^{(\rho+1) h} f\left(x q^{h}\right) \\
& \times \sum_{p=0}^{\infty}\left\{\frac{\left(q^{-M_{1}} ; q\right)_{p} \prod_{u=1}^{r-1}\left(q^{\alpha_{u}} ; q\right)_{A_{u} p}}{(q ; q)_{p}\left(q^{1-p+M_{1}} ; q\right)_{1+M_{2}} \prod_{u=1}^{s}\left(q^{\beta_{u}} ; q\right)_{B_{u} p}}\right. \\
& \times\left[(1-q)^{p} q^{\left(\begin{array}{c}
p \\
2
\end{array}\right)}\right]^{\sum_{u=1}^{s} B_{u}-\sum_{u=1}^{r-1} A_{u}} \\
& \frac{\left(q^{b_{12}+m_{12}-1-M_{1}}, \ldots, q^{b_{s 2}+m_{s 2}-1-M_{1}} ; q\right)_{p}}{\left(q^{b_{12}-1-M_{1}}, \ldots, q^{b_{s 2}-1-M_{1}} ; q\right)_{p}} q^{\left(n+h-M_{2}\right) p} .
\end{aligned}
$$

Following to Galué [15], p.47,

$$
\left(q^{1-p+M_{1}} ; q\right)_{1+M_{2}}=\frac{(q ; q)_{M_{2}+M_{1}+1}}{(q ; q)_{M_{1}}} \frac{\left(q^{-M_{1}} ; q\right)_{p}}{\left(q^{-M_{2}-M_{1}-1} ; q\right)_{p}} q^{-p\left(M_{2}+1\right)} .
$$

The substitution of this result in (44) yields

$$
\begin{aligned}
& L_{q}(\cdot) I_{q}^{n}[\cdot] f(x)= \frac{(1-q)^{2}}{\Gamma_{q}\left(M_{1}+1\right) \Gamma_{q}\left(M_{2}+1\right)} \\
& \times \prod_{v=1}^{s}\left[\frac{\Gamma_{q}\left(b_{v 2}+m_{v 2}-1-M_{1}\right) \Gamma_{q}\left(b_{v 2}\right)}{\Gamma_{q}\left(b_{v 2}-1-M_{1}\right) \Gamma_{q}\left(b_{v 2}+m_{v 2}\right)}\right] \\
& \times q^{\left(1+M_{1}\right) M_{2}} \frac{(q ; q)_{M_{1}}(q ; q)_{M_{2}}}{(q ; q)_{M_{2}+M_{1}+1}} \\
& \times \sum_{h=0}^{\infty} q^{(\rho+1) h} f\left(x q^{h}\right) \sum_{p=0}^{\infty} \frac{\left(q^{-M_{2}-M_{1}-1} ; q\right)_{p} \prod_{u=1}^{r-1}\left(q^{\alpha_{u}} ; q\right)_{A_{u} p}}{(q ; q)_{p} \prod_{u=1}^{s}\left(q^{\beta_{u}} ; q\right)_{B_{u} p}}
\end{aligned}
$$




$$
\begin{aligned}
& \times \frac{\left(q^{b_{12}+m_{12}-1-M_{1}}, \ldots, q^{b_{s 2}+m_{s 2}-1-M_{1}} ; q\right)_{p}}{\left(q^{b_{12}-1-M_{1}}, \ldots, q^{b_{s 2}-1-M_{1}} ; q\right)_{p}} \\
& \times\left[(1-q)^{p} q^{\left(\begin{array}{c}
p \\
2
\end{array}\right)}\right]^{\sum_{u=1}^{s} B_{u}-\sum_{u=1}^{r-1} A_{u}} q^{(n+h+1) p} .
\end{aligned}
$$

On the other hand, applying (13) and after (14), we have

$$
\frac{(q ; q)_{M_{1}}(q ; q)_{M_{2}}}{(q ; q)_{M_{2}+M_{1}+1}}=\frac{\Gamma_{q}\left(M_{2}+1\right) \Gamma_{q}\left(M_{1}+1\right)}{(1-q) \Gamma_{q}\left(M_{1}+M_{2}+2\right)}
$$

now, taking in account this result and (20), (45) takes the form

$$
\begin{gathered}
L_{q}(\cdot) I_{q}^{n}[\cdot] f(x)=\frac{(1-q) q^{\left(1+M_{1}\right) M_{2}}}{\Gamma_{q}\left(M_{2}+M_{1}+2\right)} \\
\times \prod_{v=1}^{s}\left[\frac{\Gamma_{q}\left(b_{v 2}+m_{v 2}-1-M_{1}\right) \Gamma_{q}\left(b_{v 2}\right)}{\Gamma_{q}\left(b_{v 2}-1-M_{1}\right) \Gamma_{q}\left(b_{v 2}+m_{v 2}\right)}\right] \sum_{h=0}^{\infty} q^{(\rho+1) h} \\
\times_{r+s} \psi_{2 s}^{*}\left[\begin{array}{l}
\left(\alpha_{1}, A_{1}\right), \ldots,\left(\alpha_{r-1}, A_{r-1}\right),\left(b_{12}+m_{12}-1-M_{1}, 1\right), \ldots, \\
\left(\beta_{1}, B_{1}\right), \ldots,\left(\beta_{s}, B_{s}\right),
\end{array}\right. \\
\left.\left(b_{s 2}+m_{s 2}-1-M_{1}, 1\right),\left(q^{-\left(M_{2}+M_{1}+1\right)}, 1\right) \mid q, q^{n+1+h}\right] f\left(x q^{h}\right) . \\
\left(b_{12}-1-M_{1}, 1\right), \ldots,\left(b_{s 2}-1-M_{1}, 1\right)
\end{gathered}
$$

Interpreting the above expression with the help of (32) the proof is completed.

Theorem 7. Let $f$ be a $q$-absolutely continuous function on $[0, x]$,

$$
L_{q}\left[\begin{array}{c}
M_{u}, b_{1 u}, b_{2 u}, \ldots, b_{s u} \\
\rho, m_{1 u}, m_{2 u}, \ldots, m_{s u}
\end{array}\right](\cdot) \text { and } I_{q}^{n}\left[\begin{array}{l}
\rho,(M, 1),\left(\alpha_{i}, A_{i}\right)_{1, r-1} \\
\left(\beta_{j}, B_{j}\right)_{1, s}
\end{array}\right](\cdot)
$$

be fractional $q$-integral operators as defined in (8) and (31) respectively, then

$$
\begin{aligned}
L_{q}\left[\begin{array}{l}
M_{u}, b_{1 u}, b_{2 u}, \ldots, b_{s u} \\
\rho+M_{1}+M_{2}+\ldots+M_{u-1}+u-1, m_{1 u}, m_{2 u}, \ldots, m_{s u}
\end{array}\right] \\
\times L_{q}\left[\begin{array}{l}
M_{u-1}, b_{1 u-1}, b_{2 u-1}, \ldots, b_{s u-1} \\
\rho+M_{1}+M_{2}+\ldots+M_{u-2}+u-2, m_{1 u-1}, m_{2 u-1}, \ldots, m_{s u-1}
\end{array}\right] \\
\ldots L_{q}\left[\begin{array}{l}
M_{2}, b_{12}, b_{22}, \cdots, b_{s 2} \\
\rho+M_{1}+1, m_{12}, m_{22}, \cdots, m_{s 2}
\end{array}\right]
\end{aligned}
$$




$$
\begin{aligned}
& \times I_{q}^{n}\left[\begin{array}{l}
\rho,\left(M_{1}, 1\right),\left(\alpha_{i}, A_{i}\right)_{1, r-1} \\
\left(\beta_{j}, B_{j}\right)_{1, s}
\end{array}\right] f(x) \\
& =\left\{\prod_{h=2}^{u} \prod_{v=1}^{s} \frac{\Gamma_{q}\left(b_{v h}+m_{v h}-\left(\sum_{k=1}^{h-1} M_{k}+h-1\right)\right) \Gamma_{q}\left(b_{v h}\right)}{\Gamma_{q}\left(b_{v h}-\left(\sum_{k=1}^{h-1} M_{k}+h-1\right)\right) \Gamma_{q}\left(b_{v h}+m_{v h}\right)}\right. \\
& \left.\times q^{\left(h-1+\sum_{k=1}^{h-1} M_{k}\right) M_{h}}\right\} \\
& I_{q}^{n+u-1}\left[\begin{array}{l}
\rho,\left(M_{u}+M_{u-1}+\ldots+M_{1}+u-1,1\right),\left(\alpha_{i}, A_{i}\right)_{1, r-1}, \\
\left(\beta_{j}, B_{j}\right)_{1, s},\left(b_{j 2}-1-M_{1}, 1\right)_{1, s},
\end{array}\right. \\
& \left(b_{j 2}+m_{j 2}-1-M_{1}, 1\right)_{1, s},\left(b_{j 3}+m_{j 3}-2-M_{1}-M_{2}, 1\right)_{1, s}, \ldots, \\
& \left(b_{j 3}-2-M_{1}-M_{2}, 1\right)_{1, s}, \ldots \text {, } \\
& \left.\begin{array}{l}
\left(b_{j u}+m_{j u}-u+1-M_{1}-M_{2}-\cdots-M_{u-1}, 1\right)_{1, s} \\
\left(b_{j u}-u+1-M_{1}-M_{2}-\cdots-M_{u-1}, 1\right)_{1, s}
\end{array}\right] f(x), \\
& A_{i}, B_{j} \in \mathbb{R}^{+}, \operatorname{Re}\left(\alpha_{i}\right)>0, \operatorname{Re}\left(\beta_{j}\right)>0, i=1, \ldots, r-1 ; j=1, \ldots, s, \\
& M_{1}, \ldots, M_{u}, m_{j 2}, \ldots, m_{j u} \in \mathbb{N}_{0}, n \in \mathbb{N}, \rho \in \mathbb{C}, \quad 0<q<1, \\
& \sum_{j=1}^{s} B_{j}-\sum_{i=1}^{r-1} A_{i} \geq 0, b_{v u} \neq u-1+M_{1}+M_{2}+M_{u-1}, \\
& u-2+M_{1}+M_{2}+M_{u-1}, u-3+M_{1}+M_{2}+M_{u-1}, \ldots, \\
& M_{u} \geq m_{1 u}+\ldots+m_{s u}, v=1, \ldots, s, u=2,3, \ldots
\end{aligned}
$$

Proof. To prove (47), we employ the mathematical induction principle.

We observe that for $u=2$ in (47), it yields to eq. (40).

Now, we suppose that eq. (47) is true for $u=l>2$, that is,

$$
\begin{gathered}
L_{q}\left[\begin{array}{l}
M_{l}, b_{l}, b_{2 l}, \ldots, b_{s l} \\
\rho+M_{1}+M_{2}+\ldots+M_{l-1}+l-1, m_{1 l}, m_{2 l}, \ldots, m_{s l}
\end{array}\right] \\
\times L_{q}\left[\begin{array}{l}
M_{l-1}, b_{1 l-1}, b_{2 l-1}, \ldots, b_{s l-1} \\
\rho+M_{1}+M_{2}+\ldots+M_{l-2}+l-2, m_{1 l-1}, m_{2 l-1}, \ldots, m_{s l-1}
\end{array}\right] \\
\times \ldots L_{q}\left[\begin{array}{l}
M_{2}, b_{12}, b_{22}, \cdots, b_{s 2} \\
\rho+M_{1}+1, m_{12}, m_{22}, \cdots, m_{s 2}
\end{array}\right] \\
\times I_{q}^{n}\left[\begin{array}{l}
\rho,\left(M_{1}, 1\right),\left(\alpha_{i}, A_{i}\right)_{1, r-1} \\
\left(\beta_{j}, B_{j}\right)_{1, s}
\end{array}\right] f(x)
\end{gathered}
$$




$$
\begin{aligned}
& \left\{\prod_{h=2}^{l} \prod_{v=1}^{s} \frac{\Gamma_{q}\left(b_{v h}+m_{v h}-\left(\sum_{k=1}^{h-1} M_{k}+h-1\right)\right) \Gamma_{q}\left(b_{v h}\right)}{\Gamma_{q}\left(b_{v h}-\left(\sum_{k=1}^{h-1} M_{k}+h-1\right)\right) \Gamma_{q}\left(b_{v h}+m_{v h}\right)}\right. \\
& \left.\times q^{\left(h-1+\sum_{k=1}^{h-1} M_{k}\right) M_{h}}\right\} \\
& \times I_{q}^{n+l-1}\left[\begin{array}{l}
\rho,\left(M_{l}+M_{l-1}+\ldots+M_{1}+l-1,1\right),\left(\alpha_{i}, A_{i}\right)_{1, r-1}, \\
\left(\beta_{j}, B_{j}\right)_{1, s},\left(b_{j 2}-1-M_{1}, 1\right)_{1, s},
\end{array}\right. \\
& \left(b_{j 2}+m_{j 2}-1-M_{1}, 1\right)_{1, s},\left(b_{j 3}+m_{j 3}-2-M_{1}-M_{2}, 1\right)_{1, s}, \ldots, \\
& \left(b_{j 3}-2-M_{1}-M_{2}, 1\right)_{1, s}, \ldots, \\
& \left.\begin{array}{l}
\left(b_{j l}+m_{j l}-l+1-M_{1}-M_{2}-\cdots-M_{l-1}, 1\right)_{1, s} \\
\left(b_{j l}-l+1-M_{1}-M_{2}-\cdots-M_{l-1}, 1\right)_{1, s}
\end{array}\right] f(x),
\end{aligned}
$$

$A_{i}, B_{j} \in \mathbb{R}^{+}, \operatorname{Re}\left(\alpha_{i}\right)>0, \operatorname{Re}\left(\beta_{j}\right)>0, i=1, \ldots, r-1, j=1, \ldots, s$,

$M_{1}, \ldots, M_{l}, m_{j 2}, \ldots m_{j l} \in \mathbb{N}_{0}, n \in \mathbb{N}, \rho \in \mathbb{C}, 0<q<1$,

$\sum_{j=1}^{s} B_{j}-\sum_{i=1}^{r-1} A_{i} \geq 0, b_{v l} \neq l-1+M_{1}+M_{2}+\ldots+M_{l-1}$,

$$
\begin{gathered}
l-2+M_{1}+M_{2}+\ldots+M_{l-1}, l-3+M_{1}+M_{2}+\ldots+M_{l-1}, \ldots, \\
M_{l} \geq m_{1 l}+\ldots+m_{s l}, v=1, \ldots, s, l=3,4, \ldots
\end{gathered}
$$

On operating both sides of (48) by the operator

$$
L_{q}\left[\begin{array}{l}
M_{l+1}, b_{1 l+1}, b_{2 l+1}, \ldots, b_{s l+1} \\
\rho+M_{1}+M_{2}+\ldots+M_{l}+l, m_{1 l+1}, m_{2 l+1}, \ldots, m_{s l+1}
\end{array}\right](\cdot),
$$

we have

$$
\begin{gathered}
L_{q}\left[\begin{array}{l}
M_{l+1}, b_{1 l+1}, b_{2 l+1}, \ldots, b_{s l+1} \\
\rho+M_{1}+M_{2}+\ldots+M_{l}+l, m_{1 l+1}, m_{2 l+1}, \ldots, m_{s l+1}
\end{array}\right] \\
\times L_{q}\left[\begin{array}{l}
M_{l}, b_{l}, b_{2 l}, \ldots, b_{s l} \\
\rho+M_{1}+M_{2}+\ldots+M_{l-1}+l-1, m_{1 l}, m_{2 l}, \ldots, m_{s l}
\end{array}\right] \\
\times L_{q}\left[\begin{array}{l}
M_{l-1}, b_{1 l-1}, b_{2 l-1}, \ldots, b_{s l-1} \\
\rho+M_{1}+M_{2}+\ldots+M_{l-2}+l-2, m_{1 l-1}, m_{2 l-1}, \ldots, m_{s l-1}
\end{array}\right] \ldots \\
L_{q}\left[\begin{array}{l}
M_{2}, b_{12}, b_{22}, \ldots, b_{s 2} \\
\rho+M_{1}+1, m_{12}, m_{22}, \cdots, m_{s 2}
\end{array}\right] \\
I_{q}^{n}\left[\begin{array}{l}
\rho,\left(M_{1}, 1\right),\left(\alpha_{i}, A_{i}\right)_{1, r-1} \\
\left(\beta_{j}, B_{j}\right)_{1, s}
\end{array}\right] f(x)
\end{gathered}
$$




$$
\begin{aligned}
& =\left\{\prod_{h=2}^{l} \prod_{v=1}^{s} \frac{\Gamma_{q}\left(b_{v h}+m_{v h}-\left(\sum_{k=1}^{h-1} M_{k}+h-1\right)\right) \Gamma_{q}\left(b_{v h}\right)}{\Gamma_{q}\left(b_{v h}-\left(\sum_{k=1}^{h-1} M_{k}+h-1\right)\right) \Gamma_{q}\left(b_{v h}+m_{v h}\right)}\right. \\
& \left.\times q^{\left(h-1+\sum_{k=1}^{h-1} M_{k}\right) M_{h}}\right\} \\
& \times L_{q}\left[\begin{array}{l}
M_{l+1}, b_{1 l+1}, b_{2 l+1}, \ldots, b_{s l+1} \\
\rho+M_{1}+M_{2}+\ldots+M_{l}+l, m_{1 l+1}, m_{2 l+1}, \ldots, m_{s l+1}
\end{array}\right] \\
& \times I_{q}^{n+l-1}\left[\begin{array}{l}
\rho,\left(M_{l}+M_{l-1}+\ldots+M_{1}+l-1,1\right),\left(\alpha_{i}, A_{i}\right)_{1, r-1}, \\
\left(\beta_{j}, B_{j}\right)_{1, s},\left(b_{j 2}-1-M_{1}, 1\right)_{1, s},
\end{array}\right. \\
& \left(b_{j 2}+m_{j 2}-1-M_{1}, 1\right)_{1, s},\left(b_{j 3}+m_{j 3}-2-M_{1}-M_{2}, 1\right)_{1, s}, \ldots, \\
& \left(b_{j 3}-2-M_{1}-M_{2}, 1\right)_{1, s}, \ldots \text {, } \\
& \left.\begin{array}{l}
\left(b_{j l}+m_{j l}-l+1-M_{1}-M_{2}-\cdots-M_{l-1}, 1\right)_{1, s} \\
\left(b_{j l}-l+1-M_{1}-M_{2}-\cdots-M_{l-1}, 1\right)_{1, s}
\end{array}\right] f(x)
\end{aligned}
$$

$A_{i}, B_{j} \in \mathbb{R}^{+}, \operatorname{Re}\left(\alpha_{i}\right)>0, \operatorname{Re}\left(\beta_{j}\right)>0, i=1, \ldots, r-1, j=1, \ldots, s$,

$$
M_{1}, \ldots, M_{l+1}, m_{j 2}, \ldots m_{j l+1} \in \mathbb{N}_{0}, n \in \mathbb{N}, \rho \in \mathbb{C}, 0<q<1,
$$

$$
\begin{gathered}
\sum_{j=1}^{s} B_{j}-\sum_{i=1}^{r-1} A_{i} \geq 0, b_{v l+1} \neq l+M_{1}+M_{2}+\ldots+M_{l}, \\
l-1+M_{1}+M_{2}+\ldots+M_{l}, l-2+M_{1}+M_{2}+\ldots+M_{l}, \ldots, \\
M_{l+1} \geq m_{1 l+1}+\ldots+m_{s l+1}, v=1, \ldots, s, l=3,4, \ldots
\end{gathered}
$$

By applying (40) to the right hand side of (49) we obtain

$$
\begin{gathered}
L_{q}\left[\begin{array}{l}
M_{l+1}, b_{1 l+1}, b_{2 l+1}, \ldots, b_{s l+1} \\
\rho+M_{1}+M_{2}+\ldots+M_{l}+l, m_{1 l+1}, m_{2 l+1}, \ldots, m_{s l+1}
\end{array}\right] \\
\times L_{q}\left[\begin{array}{l}
M_{l}, b_{l}, b_{2 l}, \ldots, b_{s l} \\
\rho+M_{1}+M_{2}+\ldots+M_{l-1}+l-1, m_{1 l}, m_{2 l}, \ldots, m_{s l}
\end{array}\right] \\
\times L_{q}\left[\begin{array}{l}
M_{l-1}, b_{1 l-1}, b_{2 l-1}, \ldots, b_{s l-1} \\
\rho+M_{1}+M_{2}+\ldots+M_{l-2}+l-2, m_{1 l-1}, m_{2 l-1}, \ldots, m_{s l-1}
\end{array}\right] \ldots \\
L_{q}\left[\begin{array}{l}
M_{2}, b_{12}, b_{22}, \ldots, b_{s 2} \\
\rho+M_{1}+1, m_{12}, m_{22}, \cdots, m_{s 2}
\end{array}\right] \\
\times I_{q}^{n}\left[\begin{array}{l}
\rho,\left(M_{1}, 1\right),\left(\alpha_{i}, A_{i}\right)_{1, r-1} \\
\left(\beta_{j}, B_{j}\right)_{1, s}
\end{array}\right] f(x)
\end{gathered}
$$




$$
\begin{aligned}
& =\left\{\prod_{h=2}^{l+1} \prod_{v=1}^{s} \frac{\Gamma_{q}\left(b_{v h}+m_{v h}-\left(\sum_{k=1}^{h-1} M_{k}+h-1\right)\right) \Gamma_{q}\left(b_{v h}\right)}{\Gamma_{q}\left(b_{v h}-\left(\sum_{k=1}^{h-1} M_{k}+h-1\right)\right) \Gamma_{q}\left(b_{v h}+m_{v h}\right)}\right. \\
& \left.\times q^{\left(h-1+\sum_{k=1}^{h-1} M_{k}\right) M_{h}}\right\} \\
& L_{q}\left[\begin{array}{l}
M_{l+1}, b_{1 l+1}, b_{2 l+1}, \ldots, b_{s l+1} \\
\rho+M_{1}+M_{2}+\ldots+M_{l}+l, m_{1 l+1}, m_{2 l+1}, \ldots, m_{s l+1}
\end{array}\right] \\
& \times I_{q}^{n+l}\left[\begin{array}{l}
\rho,\left(M_{l+1}+M_{l}+\ldots+M_{1}+l, 1\right),\left(\alpha_{i}, A_{i}\right)_{1, r-1}, \\
\left(\beta_{j}, B_{j}\right)_{1, s},\left(b_{j 2}-1-M_{1}, 1\right)_{1, s},
\end{array}\right. \\
& \left(b_{j 2}+m_{j 2}-1-M_{1}, 1\right)_{1, s},\left(b_{j 2}-1-M_{1}, 1\right)_{1, s}, \\
& \left(b_{j 3}-2-M_{1}-M_{2}, 1\right)_{1, s}, \ldots \text {, } \\
& \left(b_{j 3}+m_{j 3}-2-M_{1}-M_{2}, 1\right)_{1, s}, \ldots, \\
& \left(b_{j l+1}+m_{j l+1}-l-M_{1}-M_{2}-\ldots-M_{l}, 1\right)_{1, s},\left(b_{j l}-l+1-M_{1}-M_{2}-\ldots\right. \\
& \left.\begin{array}{l}
\left(b_{j l}+m_{j l}-l+1-M_{1}-M_{2}-\cdots-M_{l-1}, 1\right)_{1, s}, \\
\left.-M_{l-1}, 1\right)_{1, s},\left(b_{j l+1}-l-M_{1}-M_{2}-\ldots-M_{l}, 1\right)_{1, s}
\end{array}\right] f(x),
\end{aligned}
$$

$$
A_{i}, B_{j} \in \mathbb{R}^{+}, \operatorname{Re}\left(\alpha_{i}\right)>0, \operatorname{Re}\left(\beta_{j}\right)>0, i=1, \ldots, r-1, j=1, \ldots, s,
$$

$$
M_{1}, \ldots, M_{l+1}, m_{j 2}, \ldots m_{j l+1} \in \mathbb{N}_{0}, n \in \mathbb{N}, \rho \in \mathbb{C}, 0<q<1,
$$

$$
\begin{gathered}
\sum_{j=1}^{s} B_{j}-\sum_{i=1}^{r-1} A_{i} \geq 0, b_{v l+1} \neq l+M_{1}+M_{2}+\ldots+M_{l}, \\
l-1+M_{1}+M_{2}+\ldots+M_{l}, l-2+M_{1}+M_{2}+\ldots+M_{l}, \ldots, \\
M_{l+1} \geq m_{1 l+1}+\ldots+m_{s l+1}, v=1, \ldots, s, l=3,4, \ldots,
\end{gathered}
$$

Which is true for $j=l+1$. This completes the proof.

For $r=s+1, A_{i}=B_{i}=1, \alpha_{i}=b_{i}+m_{i}, \beta_{i}=b_{i}, m_{i} \in \mathbb{N}_{0} ; i=1, \cdots, s$ in (47), and using (33) we obtain the following corollary.

Corollary 8. Let $f$ be a $q$-absolutely continuous function on $[0, x]$,

$$
L_{q}\left[\begin{array}{c}
M, b_{1 u}, b_{2 u}, \ldots, b_{s u} \\
m_{1 u}, m_{2 u}, \ldots, m_{s u}
\end{array}\right](\cdot) \text { and } L_{q}^{n}\left[\begin{array}{c}
M_{1}, b_{11}, b_{21}, \ldots, b_{s 1} \\
\rho, m_{11}, m_{21}, \ldots, m_{s 1}
\end{array}\right](\cdot)
$$

be fractional $q$-integral operators as defined in (8) and (9) respectively, then

$$
L_{q}\left[\begin{array}{l}
M_{u}, b_{1 u}, b_{2 u}, \ldots, b_{s u} \\
\rho+M_{1}+M_{2}+\ldots+M_{u-1}+u-1, m_{1 u}, m_{2 u}, \ldots, m_{s u}
\end{array}\right]
$$




$$
\begin{aligned}
& L_{q}\left[\begin{array}{l}
M_{u-1}, b_{1 u-1}, b_{2 u-1}, \ldots, b_{s u-1} \\
\rho+M_{1}+M_{2}+\ldots+M_{u-2}+u-2, m_{1 u-1}, m_{2 u-1}, \ldots, m_{s u-1}
\end{array}\right] \ldots \\
& L_{q}\left[\begin{array}{l}
M_{2}, b_{12}, b_{22}, \ldots, b_{s 2} \\
\rho+M_{1}+1, m_{12}, m_{22}, \ldots, m_{s 2}
\end{array}\right] L_{q}^{n}\left[\begin{array}{l}
M_{1}, b_{11}, b_{21}, \ldots, b_{s 1} \\
\rho, m_{11}, m_{21}, \ldots, m_{s 1}
\end{array}\right] f(x) \\
& =\left\{\prod_{h=2}^{u} \prod_{v=1}^{s} \frac{\Gamma_{q}\left(b_{v h}+m_{v h}-\left(\sum_{k=1}^{h-1} M_{k}+h-1\right)\right) \Gamma_{q}\left(b_{v h}\right)}{\Gamma_{q}\left(b_{v h}-\left(\sum_{k=1}^{h-1} M_{k}+h-1\right)\right) \Gamma_{q}\left(b_{v h}+m_{v h}\right)}\right. \\
& \left.\times q^{\left(h-1+\sum_{k=1}^{h-1} M_{k}\right) M_{h}}\right\} \\
& \times L_{q}^{n+u-1}\left[\begin{array}{l}
M_{u}+M_{u-1}+\ldots+M_{1}+u-1, b_{11}, b_{21}, \ldots, b_{s 1}, b_{12}-1-M_{1}, \\
\rho, m_{11}, m_{21}, \ldots, m_{s 1}
\end{array}\right. \\
& \ldots, b_{s 2}-1-M_{1}, b_{13}-2-M_{1}-M_{2}, \ldots, b_{s 3}-2-M_{1}-M_{2}, \ldots, b_{1 u}- \\
& m_{12}, m_{22}, \ldots, m_{s 2}, m_{13}, m_{23}, \ldots, m_{s 3}, \ldots \text {, } \\
& \left.\begin{array}{l}
-u+1-M_{1}-M_{2}-\ldots-M_{u-1}, \ldots, b_{s u}-u+1-M_{1}-M_{2}-\ldots-M_{u-1} \\
m_{1 u}, \ldots, m_{s u}
\end{array}\right] \\
& \times f(x), \\
& R e\left(b_{j 1}\right) \neq 0,-1,-2, \ldots, j=1, \ldots, s, M_{1}, \ldots, M_{u}, m_{j 2}, \ldots, m_{j u} \in \mathbb{N}_{0} \\
& n \in \mathbb{N}, \rho \in \mathbb{C}, 0<q<1, b_{v u} \neq u-1+M_{1}+M_{2}+M_{u-1}, \\
& u-2+M_{1}+M_{2}+M_{u-1}, u-3+M_{1}+M_{2}+M_{u-1}, \ldots, M_{u} \geq m_{1 u}+\ldots+m_{s u}, \\
& v=1, \ldots, s, u=2,3, \ldots
\end{aligned}
$$

The result established in [15] is for the case $b_{j 1} \in \mathbb{R}, j=1, \ldots, s$.

\section{Acknowledgements}

The authors would like to thanks to Centro de Investigaciones de la Universidad de la Guajira - Colombia and to CONDES - Universidad del Zulia - Venezuela for financial support. 


\section{References}

[1] R.P. Agarwal, Certain fractional $q$-integral and $q$-derivatives, Proc. Camb. Phil. Soc., 66 (1969), 365-370.

[2] W.A. Al-Salam, Some fractional $q$-integrals and $q$-derivatives, Proc. Edin. Math. Soc., 15 (1966), 135-140.

[3] G.E. Andrews, q-Series: Their Development and Application in Analysis, Number Theory, Combinatorics, Physics, and Computer Algebra, Regional Conference Series in Math. 66, Amer. Math. Soc. (1986).

[4] M.H. Annaby, Z.S. Mansour, q-Fractional Calculus and Equations, Lecture Notes in Mathematics 2056, Springer, Berlin (2012).

[5] G. Bangerezako, Variational calculus on $q$-nonuniform lattices, J. Math. Anal. and Appl., 306 (2005), 161-179.

[6] F. Bouzaffour, Hardy type inequalities for fractional and $q$-fractional integral operators, Math. Inequal. Appl., 16 (2013), 601-609.

[7] K. Brahim and S. Taf, On some fractional $q$-integral inequalities, J. Mat., 3 (2013), 21-26.

[8] K. Brahim and S. Taf, Some fractional integral inequalities in quantum calculus, J. Fract. Calc. Appl., 4 (2013), 245-250.

[9] Y.Q. Chen and B.M. Vinagre, A new IIR-type digital fractional order differentiator, Signal Processing, 83 (2003), 2359-2365.

[10] Z. Dahmani and A. Benzidane, New weighted Gruss type inequalities via $(\alpha, \beta)$ fractional $q$-integral inequalities, Intern. J. Innovation and Appl. Studies, 1 (2012), 76-83.

[11] M. Delgado and L. Galué, Fractional $q$-integral operator involving basic hypergeometric function, Groups Geom., 25 (2008), 53-74.

[12] Y. Ding, S.Z. Shan Zhen Lu and K. Yabuta, Multilinear singular and fractional integrals, Acta Math. Sinica, 22 (2006), 347-356.

[13] L. Galué, Generalized Erdérlyi-Kober fractional $q$ - integral operator, Kuwait J. Sci. Eng., 36 (2009), 21-34.

[14] L. Galué, Generalized Weyl fractional q-integral operator, Algebras Groups and Geometries, 26 (2009), 163-178. 
[15] L. Galué, On composition of fractional $q$-integral operators involving basic hipergeometric functions, J. Ineq. and Spec. Functions, 1 (2010), 39-52.

[16] L. Galué, Unification of $q$-fractional integral operator, Algebras, Groups and Geom., 28 (2011), 283-298.

[17] L. Galué, Unified fractional $q$-integral operator of $q$-special functions, $A l$ gebras, Groups and Geom., 28 (2011), 185-204.

[18] L. Galué, Some results involving generalized Erdé lyi-Kober fractional qintegral operators, Revista Tecnocientífica URU 6 (2014), 77-89.

[19] M. Garg and L. Chanchlani, Kober fractional $q$-derivative operators, Le Matematiche 66 (2011), 13-26.

[20] M. Garg and L. Chanchlani, q-analogues of Saigo's fractional calculus operators, Bull. Math. Anal. and Appl., 3 (2011), 169-179.

[21] G. Gasper, M. Rahman, Basic Hypergeometric Series, Cambridge University Press, Cambridge (2004).

[22] H. Gauchman, Integral Inequalities in q-Calculus, Computers and Mathematics with Applications 47 (2004), 281-300.

[23] O. Gorosito, G. Pradolini and O. Salinas, Weighted weak-type estimates for multilinear commutators of fractional integrals on spaces of homogeneous type, Acta Math. Sinica, 23 (2007), 1813-1826.

[24] T. Hayat, S. Nadeem and S. Asghar, Periodic unidirectional fows of a viscoelastic fluid with the fractional Maxwell model, Appl. Math. Comput., 151 (2004), 153-161.

[25] M. Ismail, Classical and Quantum Orthogonal Polynomials in One Variables, Cambridge University Press, Cambridge (2005).

[26] F.H. Jackson, On q-definite integrals, Quart. J. Pure Appl. Math., 41 (1910), 193-203.

[27] V. Kac and P. Cheung, Quantum Calculus, Universitext, Springer, New York (2002).

[28] V. Kiryakova, Generalized Fractional Calculus and Applications, Longman - J. Wiley, Harlow - N. York (1994). 
[29] E. Koelink, Quantum Groups and q-Special Functions, Report 96-10, Universiteit van Amsterdam (1996).

[30] S. Momani and Z. Odibat, Numerical approach to differential equations of fractional order, J. Comput. Appl. Math., 207 (2007), 96-110.

[31] K.B. Oldham and J. Spanier, The Fractional Calculus, Academic Press, New York (1974).

[32] I. Podlubny, Fractional Differential Equations, Academic Press, New York (1999).

[33] S.D. Purohit and R.K. Yadav, On generalized fractional $q$-integral operators envolving the $q$-Gauss hypergeometric function, Bull. Math. Anal. Appl., 2 (2010), 35-44.

[34] S.D. Purohit and R.K. Raina, Chebyshev type inequalities for the Saigo fractional integrals and their q-analogues, J. Math. Ineq., 7 (2013), 239249.

[35] S.D. Purohit, F. Uçar, and R.K. Yadav, On fractional integral inequalities and their q-analogues, Revista Tecnocient ifica URU, 6 (2014), 53-66.

[36] E.D. Rainville, Special Functions, The MacMillan (1960).

[37] P.M. Rajkovic, S.D. Marinkovic and M.S. Stankovic, Fractional integrals and derivatives in q-calculus, Appl. Anal. Discrete Math., 1 (2007), 311323.

[38] P.M. Rajkovic, S.D. Marinkovic and M.S. Stankovic, A generalization of the concept of $q$-fractional integrals, Acta Math. Sinica (Engl. Ser.), 25 (2009), 1635-1646.

[39] B. Ross (Ed.), Fractional Calculus and Its Applications, Lecture Notes in Math., Springer-Verlag, New York (1975).

[40] M. Saigo, On the Hölder continuity of the generalized fractional integrals and derivatives, Math. Rep. Kyushu Univ., 12 (1980), 55-62.

[41] R.K. Saxena, On fractional integration operators, Math. Z., 96 (1967), 289-291.

[42] R.K. Saxena, R.K. Yadav, S.D. Purohit and S.L. Kalla, Kober fractional $q$-integral operator of the basic analogue of the $H$-function, Rev. Téc. Ing. Univ. Zulia, 28 (2005), 154-158. 
[43] A. Secer, S.D. Purohit, K.A. Selvakumaran and M. Bayram, A generalized $q$-Grüss inequality involving the Riemann-Liouville fractional $q$-integrals, J. Appl. Math., 2014 (2014), Art. ID 914320, 6 pp.

[44] L. Slater, Generalized Hypergeometric Functions, Cambridge University Press, London (1966).

[45] I.N. Sneddon, The use in mathematical physics of Erdé lyi-Kober operators and some of their generalizations, Fractional Calculus and Its Applications, Lecture Notes in Math. 457 (1975), 37-39.

[46] H.M. Srivastava, Per W. Karlsson, Multiple Gaussian Hypergeometric Series, John Wiley \& Sons, New York (1985).

[47] W.T. Sulaiman, On some new fractional $q$-integral inequalities, South Asian J. of Math., 2 (2012), 450-459.

[48] C.C. Tseng, Design of fractional order digital FIR differentiators, IEEE Signal Process. Letters, 8 (2001), 77-79.

[49] B.M. Vinagre, Y.Q. Chen and I. Petras, Two direct Tustin discretization methods for fractional-order differentiator and integrator, J. Franklin Institute, 340 (2003), 349-362.

[50] B.J. West, M. Bologna and P. Grigolini, Physics of Fractal Operators, Springer, New York (2002).

[51] R.K. Yadav and S.D. Purohit, Application of Riemann-Liouville fractional $q$-integral operator to basic hypergeometric functions, Acta Ciencia Indica, 30 (2004), 593-600.

[52] R.K. Yadav and S.D. Purohit, On applications of Weyl fractional qintegral operator to generalized basic hypergeometric functions, Kyungpook Math. J., 11 (2008), 129-142.

[53] K. Yao, W.Y. Su, S.P. Zhou, The fractional derivatives of a fractal function, Acta Math. Sinica, 22 (2006), 719-722.

[54] G.M. Zaslavsky, Hamiltonian Chaos and Fractional Dynamics, Oxford University Press, Oxford (2005). 